\title{
Multiscale views of an Alfvénic slow solar wind: 3D velocity distribution functions observed by the Proton-Alpha Sensor of Solar Orbiter
}

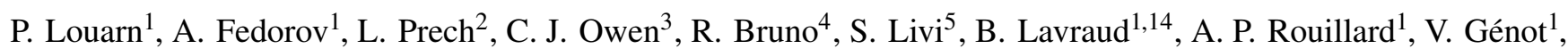
N. André ${ }^{1}$, G. Fruit ${ }^{1}$, V. Réville ${ }^{1}$, R. Kieokaew ${ }^{1}$, I. Plotnikov ${ }^{1}$, E. Penou ${ }^{1}$, A. Barthe ${ }^{6}$, D. Khataria ${ }^{3}$, M. Berthomier ${ }^{7}$, R. D’Amicis ${ }^{4}$, L. Sorriso-Valvo ${ }^{15,17}$, F. Allegrini ${ }^{5}$, J. Raines ${ }^{8}$, D. Verscharen ${ }^{3,16}$, V. Fortunato ${ }^{9}$, G. Mele ${ }^{10}$, T. S. Horbury ${ }^{11}$, H. O'brien ${ }^{11}$, V. Evans ${ }^{11}$, V. Angelini ${ }^{11}$, M. Maksimovic ${ }^{12}$, J. C. Kasper ${ }^{8}$, and S. D. Bale ${ }^{13}$

${ }^{1}$ Institut de Recherche en Astrophysique et Planétologie, CNRS, Université de Toulouse, CNES, Toulouse, France e-mail: philippe.louarn@irap.omp.eu

2 Department of Surface and Plasma Science, Faculty of Mathematics and Physics, Charles University, 18000 Prague 8, Czech Republic

3 Mullard Space Science Laboratory, University College London, Holmbury St. Mary, Dorking, Surrey RH5 6NT, UK

${ }^{4}$ INAF-Istituto di Astrofisica e Planetologia Spaziali, Via Fosso del Cavaliere 100, 00133 Roma, Italy

5 Southwest Research Institute, 6220 Culebra Road, San Antonio, TX 78238, USA

6 AKA, Toulouse, France

7 Laboratoire de Physique des Plasmas, Ecole Polytechnique, Palaiseau, France

8 Department of Climate and Space Sciences and Engineering, The University of Michigan, Ann Harbour, MI, USA

9 Planetek Italia S.r.l., Via Massaua, 12, 70132 Bari BA, Italy

10 Leonardo, Viale del lavoro, 101, 74123 Taranto, Italy

11 Space and Atmospheric Physics, The Blackett Laboratory, Imperial College London, London SW7 2AZ, UK

12 LESIA, Observatoire de Paris, Université PSL, CNRS, Sorbonne Université, Université de Paris, 5 place Jules Janssen, 92195 Meudon, France

13 Space Sciences Laboratory, University of California, Berkeley, CA 94720, USA

14 Laboratoire d'astrophysique de Bordeaux, Univ. Bordeaux, CNRS, Pessac, France

15 National Research Council, Institute for the Science and Technology of Plasmas, Via Amendola 122/D, 70126 Bari, Italy

16 Space Science Center, University of New Hampshire, 8 College Road, Durham, NH 03824, USA

17 Swedish Institute for Space Physics, Ångström Laboratory, Lägerhyddsvägen 1, 75121 Uppsala, Sweden

Received 15 April 2021 / Accepted 11 October 2021

\section{ABSTRACT}

Context. The Alfvénic slow solar wind is of particular interest, as it is often characterized by intense magnetic turbulence, complex proton 3D velocity distribution functions (VDF), and an ensuing richness of kinetic and dynamic processes.

Aims. We take advantage of the fast time cadence of measurements taken by the Proton-Alpha Sensor (PAS) on board Solar Orbiter to analyze the kinetic properties of the proton population, the variability of their VDFs, and the possible link with propagating magnetic structures. We also study the magnetic $(\mathbf{B})$ and velocity $(\mathbf{V})$ correlation that characterizes this type of wind down to the ion gyroperiod. Methods. We analyzed the VDFs measured by PAS, a novelty that take advantages of the capability of $3 \mathrm{D}$ measurements at a $4 \mathrm{~Hz}$ cadence. In addition, we considered MAG observations.

Results. We first show that there is a remarkable correlation between the $\mathbf{B}$ and $\mathbf{V}$ components observed down to timescales approaching the ion gyrofrequency. This concerns a wide variety of fluctuations, such as waves, isolated peaks, and discontinuities. The great variability of the proton VDFs is also documented. The juxtaposition of a core and a field-aligned beam is the norm but the relative density of the beam, drift speed, and temperatures can considerably change on scales as short as as a few seconds. The characteristics of the core are comparatively more stable. These variations in the beam characteristics mostly explain the variations in the total parallel temperature and, therefore, in the total anisotropy of the proton VDFs. Two magnetic structures that are associated with significant changes in the shape of VDFs, one corresponding to relaxation of total anisotropy and the other to its strong increase, are analyzed here. Our statistical analysis shows a clear link between total anisotropy (and, thus, beam characteristics) and the direction of $\mathbf{B}$ with respect to the Parker spiral. In the present case, flux tubes aligned with Parker spiral contain an average proton VDF with a much more developed beam (thus, with larger total anisotropy) than those that are inclined, perpendicular, or even reverse with regard to the outward direction.

Conclusions. These observations document the variability of the proton VDF shape in relation to the propagation of magnetic structures. This is a key area of interest for understanding of the effect of turbulence on solar wind dynamics.

Key words. solar wind - turbulence - plasmas 


\section{Introduction}

The analysis of kinetic processes in the data is an essential step in fully understanding the solar wind dynamics. They contribute to the heating and acceleration of the plasma populations, the evolution of turbulence, the formation of structures, and the generation of waves. To study them, it is essential to access to the precise kinetic organization of the plasma, thus, to measure the velocity distribution functions (VDF) of the different species, at the relevant scales. These measurements, for the proton and alpha populations, are the main objective of the Proton Alpha Sensor (PAS), part of the SWA instrument suite on board Solar Orbiter (Owen et al. 2020; Müller et al. 2020).

Concerning protons, the first 3D VDFs were obtained in the 1970s (Hundhausen 1970). It has been shown that these functions are symmetrical with respect to the magnetic field (gyrotropic function) with, however, a general asymmetry in the direction of the flow: the peak of the distribution is shifted toward lower energy outstreaming velocities and a tail extends toward higher energy. These distributions, measured with integration times of several tens of seconds due to experimental constraints, have been modeled by Bi-Maxwellian or Kappa-type distributions by Whang (1971), Cuperman et al. (1983), Demars \& Schunk (1990), Leubner (2004), Pierrard \& Lazar (2010), and for electrons by Maksimovic et al. (1997).

A major step in improving our knowledge of solar wind VDF has been accomplished thanks to the measurements of Helios probes. A seminal work was achieved by Marsch et al. (1981, 1982), to better describe the shape of the proton VDF, confirming the fact that they are organized with respect to the magnetic field direction. Close to the Sun and in the fast wind, their core generally spreads in the perpendicular to the $\mathbf{B}$ direction, such that $T_{\perp} / T_{\|}>1$, where $T_{\perp}\left(T_{\|}\right)$is the perpendicular (parallel) to $\mathbf{B}$ temperature. The field-aligned proton beams frequently occur at drift speed slightly larger than the local Alfven velocity. Doublepeak distributions, corresponding to a core plus a detached beam, are also commonly observed. Such distributions may drive various plasma instabilities and feed heating processes. A review of Helios observations and solar wind kinetic processes can be found in Marsch (2006).

Since the observations of Helios, the knowledge of the solar wind plasma and its dynamics has improved considerably with missions such as Ulysses, Wind, Stereo, but also magnetosheric missions such as Cluster, Themis, and MMS. The measurements of the "plasma" instruments of these missions have made it possible to document many aspects of the physics of the solar wind, including turbulence, ion composition, the coupling to coronal structures, the energetic events (CME, shocks... ), and multiple aspects of microphysics, down to electron scales. For reviews on these subjects, see Tu \& Marsch (1995), Marsch (2006), von Steiger (2008), Bruno \& Carbone (2013), Viall \& Borovsky (2020), Verscharen et al. (2019). Currently, the measurements carried out at distances of less than $35 R_{\odot}$ from the Sun's surface by Parker Solar Probe (PSP) are opening new chapters in our understanding of the acceleration and heating processes of the pristine solar wind (Fox et al. 2016; Bale et al. 2019; Kasper et al. 2019).

Despite the sophistication of the plasma instruments of these missions, it is notable that knowledge of 3D VDF of the solar wind protons has not fundamentally changed since the Helios observations. In this context, PAS measurements offer a novel approach. Even in normal mode (4s cadence), PAS takes an instant 3D "picture" of VDFs since most of the solar wind protons are sampled in $\sim 0.1-0.15 \mathrm{~s}$, which has never been done before. In its burst mode (rate of $0.25 \mathrm{~s}$ ), 3D measurements are carried out at unprecedented cadence,with only MMS having done better, but in the terrestrial magnetosphere.

A large part of the present article is devoted to the study of these 3D proton VDFs, measured in an Alfvénic slow solar wind. Their general characteristics are detailed as their variability and examples of structures propagating in the solar wind that modify the shape of the VDFs are presented.

The existence of Alfvénic winds is a well-established observational fact, which dates back to the first in-situ measurements of the solar wind (Belcher \& Davis 1969, 1971; Bruno et al. 1985). The slow Alfvénic wind was first described in the internal heliosphere from observations by Helios (Marsch et al. 1981). It is characterized by the strong amplitude and cross-correlation of the fluctuations of $\mathbf{B}$ and $\mathbf{V}$ vectors $\left(\delta B / B>0.5, \delta V / V_{a}>0.5\right.$, where $V_{a}$ is the Alfvén velocity). In many respects, including ion composition, relative characteristics of the various populations, and turbulence characteristics, this type of slow wind present similarities with the fast wind (Roberts et al. 1987; D'Amicis et al. 2019) and appears to be relatively common at 1 a.u. (D'Amicis et al. 2011).

In mid-July 2020, Solar Orbiter crossed a slow wind that demonstrates a particularly large level of fluctuation, while the distance to the Sun was 0.65 a.u. The characteristics of this wind as well as its connection to the Sun's surface are analyzed in D'Amicis (2021). Here, using the PAS time resolution capability, we concentrate on short-timescale processes and microphysics aspects.

Following a presentation of the instruments and describing their operation in Sect. 2, we characterize the flow at global scales, analyze the correlation between $B$ and $V$ down to second scales in Sect. 3. In Sect. 4, we describe the observed proton VDF and propose a model to fit their core+beam shape, using biMaxwellian and Kappa distributions. In Sect. 5, two examples of structures modifying the VDF shape are presented, followed by a discussion and our conclusions in Sect. 6.

\section{Instruments and data}

Our study is based on measurements provided by the Solar Wind Analyzer (SWA) suite of instruments (Owen et al. 2020) and the Magnetometer (MAG, Horbury et al. 2020) of the Solar Orbiter mission (Müller et al. 2020; Zouganelis et al. 2020). SWA consists of an Electron Analyzer System (EAS), a Proton and Alpha particle Sensor (PAS), and a Heavy Ion Sensor (HIS) served by a data processing unit (DPU). Here, we mostly discuss the proton measurements provided by PAS.

The PAS is an electrostatic analyzer designed to measure the 3D VDF of the solar wind ions without mass selection. At full sampling ("full" 3D), PAS gets matrix of counts over 96 energies, 11 azimuth, and 9 elevation angles then converted in VDF $\left(\mathrm{s}^{3} / \mathrm{m}^{6}\right)$ using the geometrical factor of the instrument, estimated from calibrations. The "full 3D" sampling consist of $200 \mathrm{eV} / \mathrm{q}$ to $20 \mathrm{keV} / \mathrm{q},-24^{\circ}$ to $+42^{\circ}$ in azimuth, $\pm 22.5^{\circ}$ in elevation, with a $6^{\circ} \times 6^{\circ}$ angular resolution. The complete energy/elevation sweeping is effected in $1 \mathrm{~s}$, which is also the acquisition time of one full 3D VDF.

Generally, the solar wind protons occupy only a reduced part of the total phase space that PAS is able to sample. Most of the time, 32 and 5 judiciously selected energies and elevations are sufficient to characterize the proton-alpha population. To optimize the sampling, a "peak tracking" procedure has been implemented to automatically select the peak of the distribution and concentrate the sampling to the interesting part of the phase 
space. This is used to accelerate the measurement cadence. This is the principle of PAS burst mode. In the following, we use a 32 energies, 7 elevations, and 11 azimuth modes that provide 3D VDF at a $0.25 \mathrm{~s}$ cadence. The normal mode has a $4 \mathrm{~s}$ cadence and use $48 \times 9 \times 11$ samplings with full 3D each $100 \mathrm{~s}$ to actualize the peak tracking. As already mentioned, the interesting part of each distribution is obtained in typically $0.1-0.15 \mathrm{~s}$, the time needed to capture the essential of the proton population.

Let us discuss the expected accuracy of the measurements. PAS has a rather large geometrical factor $\left(5.10^{-6} \mathrm{~cm}^{2} . \mathrm{sr} . \mathrm{eV} / \mathrm{eV}\right.$ per pixel) and the total number of counts for a single 3D VDF is generally larger than 2000. This can be used to estimate the statistical errors (Moore et al. 1998; Gershman \& Dorelli 2015; Nicolaou et al. 2020). For the measurements presented below, the number of counts for 1 VDF is $n \sim 4000$. Since the density $(N)$ is proportional to $n$, the statistical relative error is simply $\sigma=n^{1 / 2} / n$. This gives $\sigma \sim 1.5 \%$ or, as we will see: $\Delta N= \pm 0.22 \mathrm{~cm}^{-3}$. The error on the speed can be estimated by $\delta V / V \sim\left(T_{\text {th }} / E\right)^{1 / 2} / n^{1 / 2}$ where $T_{\text {th }}$ is the thermal energy and $E$ the mean "bulk" energy of the flow. For $T_{\text {th }}=30 \mathrm{eV}, E=1 \mathrm{keV}$ and $n=4000$, we obtain $\delta V / V \sim 0.27 \%$. The error at $3 \sigma$ is therefore $\sim 0.7 \mathrm{~cm}^{-3}$ and $\sim 3 \mathrm{~km} \mathrm{~s}^{-1}$. As we go on to show later, this is visually larger than the envelope of the noise seen in the plots of density and velocity. The PAS data are available for download online ${ }^{1}$, including the magnetic field measurements from the MAG instrument (Génot et al. 2021). Here, we used the $0.125 \mathrm{~s}$ MAG measurements.

\section{General characteristics of a slow Alfvénic flow, from hour to second timescales}

Figure 1 shows 20 hours of combined PAS and MAG observations in a slow Alfvenic solar wind. A general discussion of this episode of slow wind and its connection to Sun's structures can be found in D'Amicis (2021). This flow is characterized by a high level of magnetic $(B)$ and plasma velocity $(V)$ fluctuations, such as $\Delta B /|B|>50 \%$ and $\Delta V / V_{a}>50 \%$. This corresponds to fluctuations of $\sim 5-10 \mathrm{nT}$ and $\sim 20-50 \mathrm{~km} \mathrm{~s}^{-1}$ for the components of $B$ or $V$, on timescales from a few tens of minutes to a few seconds.

The ion energy spectrogram, from 500 to $1700 \mathrm{eV}$ is shown in Fig. 1a. During this time period, the protons are detected from $\sim 600$ to $1300 \mathrm{eV}$ and globally centered at $\sim 950 \mathrm{eV}$. The alpha population is detected above $1500 \mathrm{eV}$, typically, and only its low energy extension is occasionally seen in the spectrogram. The proton spectral peak exhibits frequent fluctuations between $\sim 700$ and $\sim 1040 \mathrm{eV}$, on timescales that can be shorter than a minute. These "rapid" fluctuations typically reach 10-20\% of the mean value of $N$ and $T\left(\sim 2 \mathrm{~cm}^{3} \sim 3 \mathrm{eV}\right)$, and $30-50 \%$ of $B$ and $V_{a}$ $\left(\sim 5 \mathrm{nT}\right.$ and $\left.\sim 20 \mathrm{~km} \mathrm{~s}^{-1}\right)$. They appear as a form of noise in the figure, however, they are much larger than the statistical errors.

An obvious characteristic of the fluctuations is the very strong correlation or, more exactly, the anti-correlation, between each component of $B$ and $V$ (Figs. 1d-f). These correlations are observed for fluctuations of varied nature: more or less regular oscillations, slow variations at scales of a few tens of minutes or abrupt discontinuities at scale of seconds. We may notice several reversals of $B_{R}$, on scales of a few minutes to a few hours, sometimes as square-like structures (for exemple: 14/07 19:00). They are systematically accompanied by increases in $V_{R}$, by $\sim 40-60 \mathrm{~km} \mathrm{~s}^{-1}$. These fluctuations present the basic characteristics of switchbacks (Balogh et al. 1999; Suess 2007; Matteini

\footnotetext{
http://amda.irap.omp.eu/
}

et al. 2006, 2013; Borovsky 2016; Horbury et al. 2018; Bale et al. 2019; Kasper et al. 2019; Dudok de Wit et al. 2020), one of which (14/07 19:20) is described in next section (see also, Fedorov 2021, for PAS observations of a switchback). The fluctuations of $|B|$ are generally less than $2 \mathrm{nT}$, meaning less than 20 $\%$ of the averaged $|B|$. A few stronger decreases of $|B|$ are nevertheless observed at scales shorter than 1 hour. This may correspond to the propagation of compressive disturbances.

Figure 1c shows the parallel and perpendicular total temperatures $\left(T_{t \perp}\right.$ and $\left.T_{t \|}\right)$. They are obtained by the direct second moment (pressure tensor) calculation using the whole proton distribution. They have to be distinguished from the core and beam temperature described later (hence, the use of subscript ' $t$ '). The examination of the VDFs will allow to interpret the apparent total anisotropy $T_{t \|} / T_{t \perp}>1$ as an effect of the juxtaposition of a core population and a field-aligned beam, the core presenting most of the time a $T_{c \perp} / T_{c \|}>1$ anisotropy, as described in Marsch (2006).

The average values of $B, N, V$, temperature $(T)$, and pressure $(P)$ are, respectively: $B \sim 12 \mathrm{nT}, N \sim 14 \mathrm{~cm}^{-3}, V \sim 430 \mathrm{~km} \mathrm{~s}^{-1}$, $T \sim 22 \mathrm{eV}$, and $P \sim 300 \mathrm{eV} \mathrm{cm}^{-3}$. The characteristic flow parameters are therefore: $V_{a} \sim 60 \mathrm{~km} \mathrm{~s}^{-1}$, proton gyrofrequency $\left(\omega_{\mathrm{ci}}=q B / M\right) \sim 1.15 \mathrm{~Hz}$, gyroperiod $\sim 5.5 \mathrm{~s}$, thermal speed $V_{\mathrm{th}} \sim$ $65 \mathrm{~km} \mathrm{~s}^{-1}$, thermal Larmor radius $\sim 55 \mathrm{~km}$, which is also about the ion inertial length $\left(\mathrm{c} / \omega_{p i}\right.$ where $\omega_{p i}$ is the ion plasma frequency) and ratio between magnetic pressure and thermal pressure $\beta \sim 1$.

The strong degree of correlation between $B$ and $V$ is studied in more detail in Fig. 2. The magnetic field is here transformed into Alfvén velocity by the formula: $b=B /\left(\mu_{0} \rho\right)^{1 / 2}$. Each of the components of $V$ and $b$ are superimposed in Figs. 2a-c (more precisely, we superimpose $V$ and $-b$ ). The $b-V$ Pearson cross-correlations for each RTN component have been computed (Fig. 2d). They are estimated every $40 \mathrm{~s}$ on sliding time windows of $10 \mathrm{~min}$. The cross-correlations exceed 0.9 for periods of several hours, however, with a lesser or no correlation at all (correlation $<0.6$ ) for intervals shorter than $\sim 1 \mathrm{~h}$. The percentage of time of correlation greater than 0.8 is $42 \%$ for the R component, $66 \%$ for $\mathrm{T}$, and $75 \%$ for $\mathrm{N}$. The percentages of time of correlation smaller than 0.6 are $37 \%, 13 \%$, and $8 \%$ for $\mathrm{R}$, T, and $\mathrm{N}$, respectively. The average correlations are $0.66(\mathrm{R}), 0.81(\mathrm{~T})$ and $0.84(\mathrm{~N})$. In general, the $\mathrm{R}$ components are less correlated than the $\mathrm{T}$ and $\mathrm{N}$ ones.

Figure 2e shows a scatter plot of $V_{T}$ and $b_{T}$ (more exactly $-b_{T}$ ) for the time interval of 14/07 12:00-24:00 UT. For the whole period, their cross-correlation is $\sim 0.94$. The overall organization along a slope $\sim 0.65$ is obvious. This is less than 1 (the expected slope for strict Alfvénic disturbances).

The spectra of the $V$ and $b$ fluctuations are presented in Fig. 2f. They are computed as the sum of the spectral densities calculated for each component, smoothed over a frequency interval of $\sim 4 \%$. The upper frequencies are $0.125 \mathrm{~Hz}$ for $V$ and $2 \mathrm{~Hz}$ for $b$. As classically reported, a power law spectrum with exponent $\sim-1.6$ is obtained in the low frequency domain (here $10^{-4}<f<0.2 \mathrm{~Hz}$ ). This is close to $-5 / 3$, thus, typical of the inertial domain described by the Kolmogorov phenomenology. The spectra of $V$ and $b$ overlap almost exactly, except in the high-frequency part $(f>0.07 \mathrm{~Hz})$ for $V$. This difference between $V$ and $b$ corresponds to a noise on the order of $2.5 \mathrm{~km} \mathrm{~s}^{-1} \mathrm{~Hz}^{-1 / 2}$. This noise results from an under-sampling of higher frequency fluctuations. Regarding the magnetic spectrum, a change of regime is observed at $\sim 0.45-0.5 \mathrm{~Hz}$, with a steeper spectrum in the high frequency domain (exponent $\sim-3.8$ ). This is indicative of the transition from the inertial to the sub-ion 


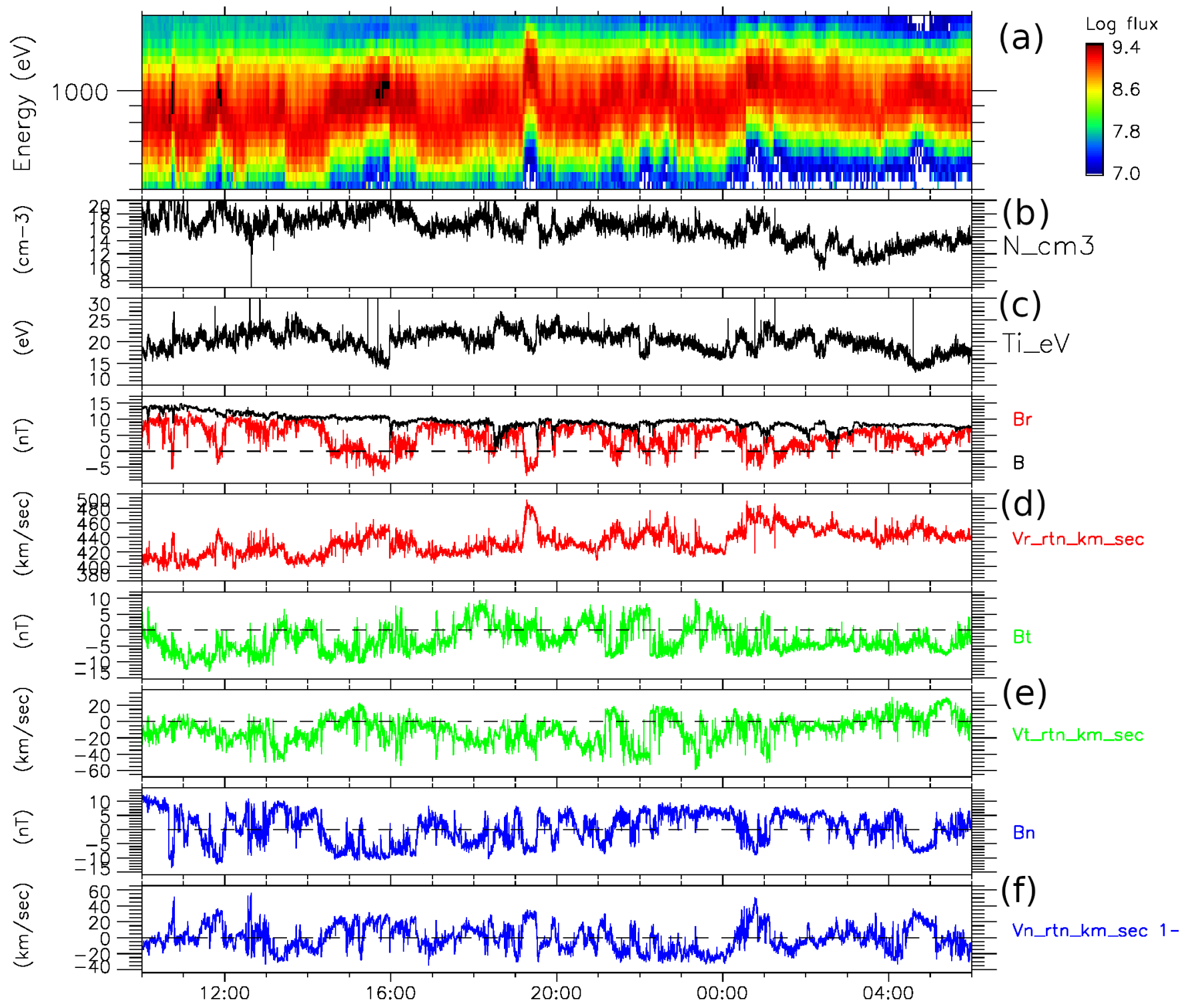

Fig. 1. PAS and MAG observations from 14/07 10:00 to 15/07 6:00. From top to bottom: (a) Time/energy spectrogram of ion flux, from 500 eV to $1.7 \mathrm{keV}$ (unit: part. $\left.\mathrm{s}^{-1} \mathrm{~cm}^{-2} \mathrm{sr}^{-1} \mathrm{keV}^{-1}\right) ;(b$ and $c$ ): proton density and temperature; $(d-f)$ : magnetic and velocity field components in RTN frame. In $(d),|\mathbf{B}|$ (in black) is also presented.

regime. It takes place at a frequency greater than the gyrofrequency $\left(f_{\text {ci }}=0.18 \mathrm{~Hz}\right)$, as expected due to the Doppler effect. The position of this frequency break is consistent with the finding of Alexandrova et al. (2009). This allows us to specify the frequency beyond which kinetic and possibly ion dissipation effect are expected (Sahraoui et al. 2009). In the rest of the paper, we consider that this transition takes place at $f \sim 0.5 \mathrm{~Hz}$ (or a period of $\sim 2 \mathrm{~s}$ ).

Figure 3 details the $b_{V}$ cross-correlation down to the ion dissipation scale. Figure 3 a presents $V$ and $b$ components ( $-b$ is shown), as well as their correlation, for a time of $1 \mathrm{~h}$. Generally speaking, the cross-correlation is greater than 0.9 for the whole interval (see Fig. 2). These excellent cross-correlations concern different types of fluctuations as, (1) progressive and simultaneous rotations of $V$ and $b$, similar to Alfven waves of long periods (around 21:40 and 22:10) and (2) discontinuities or fluctuations at scales smaller than a minute (around 22:00), with amplitudes of $30-40 \mathrm{~km} \mathrm{~s}^{-1}$. In PAS normal mode, they may not be resolved and some of these peaks correspond to a single measurement of PAS. However, these isolated peaks are not artifacts, as ensured by their independent detection with MAG. These strong fluctuations, by $\sim 30 \mathrm{~km} \mathrm{~s}^{-1}$ or $\sim 0.5 V_{a}$, are observed at scales commensurable with the ion gyroperiod and could correspond to short scale non-linear structures that are yet to be individually analyzed and identified (e.g., investigating whether they are Alfventype waves).

Figures $3 b$, c show observations made with PAS burst mode. Once again, a diversity of fluctuations is observed, in particular, regular waves of low amplitude $\left(3-5 \mathrm{~km} \mathrm{~s}^{-1}\right)$, at periods of $\sim 3-6 \mathrm{~s}$, and some isolated fluctuations of high amplitudes $\left(15-20 \mathrm{~km} \mathrm{~s}^{-1}\right.$ at $\left.t=108\right)$. Figure $3 \mathrm{c}$ shows that the $b_{V}$ crosscorrelation still exists on a timescale of seconds. In particular, from 10 to $15 \mathrm{~s}$, a wavy structure is observed both on $b$ and $V$ and perfectly resolved at the PAS burst-mode $4 \mathrm{~Hz}$ resolution. This 

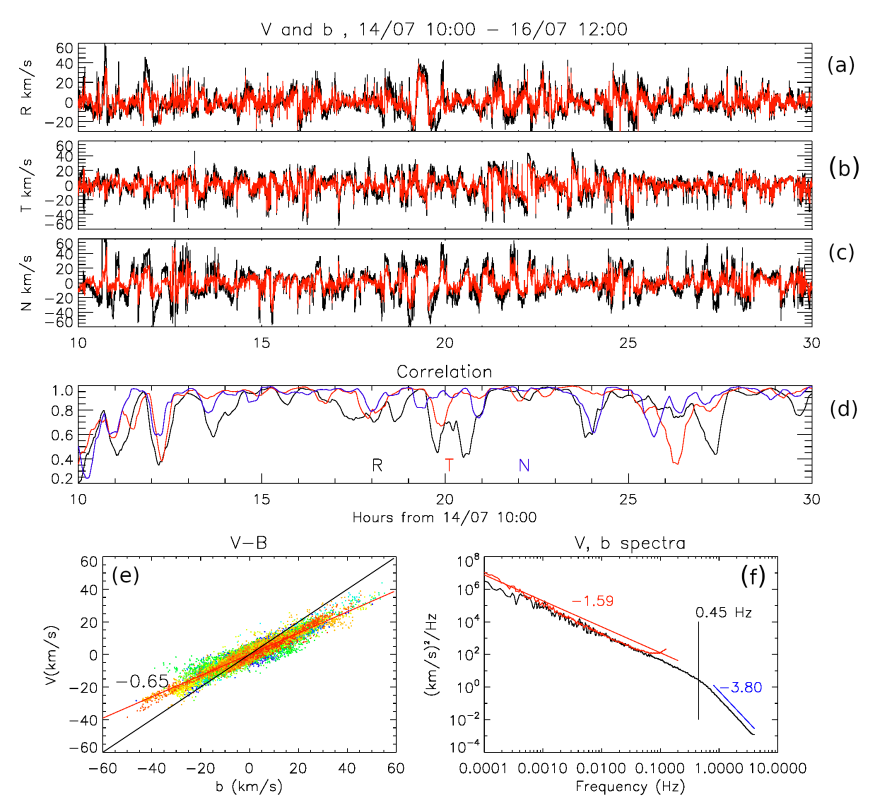

Fig. 2. $V-b$ correlation. Panels $a-c$ : RTN components of $b=B /\left(\mu_{0} \rho\right)^{1 / 2}$ (in black) and of proton velocity (in red). To better show the correlation, $-b$ is plotted. The averaged value of $V_{R}$, computed over a $1 \mathrm{~h}$ sliding window, is removed in (a). Panel $d$ : cross-correlation of R (black), T (red) and $\mathrm{N}$ (blue), calculated on ten-minute sliding windows; panel $e$ : scatter plots of $b_{T}$ and $V_{T}$, the color (from blue to red) indicates the time; panel $f$ : power spectra of $b$ (black) and $V$ (red) fluctuations.

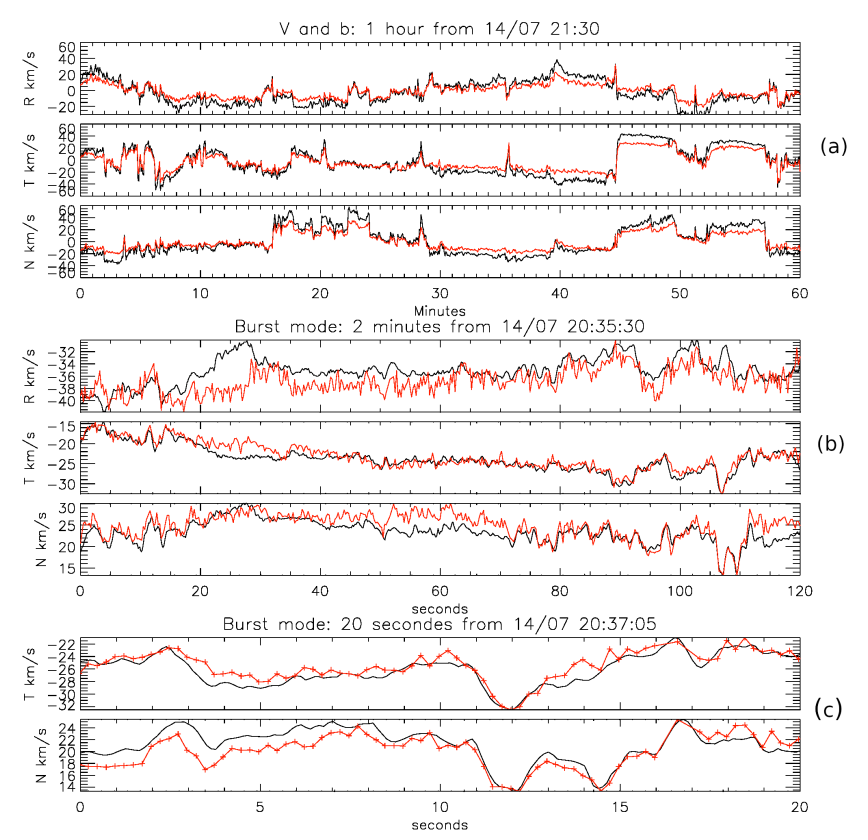

Fig. 3. $V$ (red) and $-b$ (black) correlations, from hour to second timescales. Panels $b, c$ : data obtained with PAS burst mode $(0.25 \mathrm{~s}$ cadence), compared to MAG (0.125 s cadence). The measurement points of PAS are indicated by small crosses in (c).

gives an example of $b-V$ correlation on timescale close to the ion gyroperiod. In general, the very good superposition between $V$ and $b$, at timescale of second and for fluctuations of $1-2 \mathrm{~km} \mathrm{~s}^{-1}$ (meaning $0.3 \%$ of $|V|$ ) is an indication of the excellent PAS accuracy and a form of cross-check using MAG measurements.

In summary, in this solar wind of relatively constant MHD parameters, disturbances such as $\delta B /|B|>50 \%$ and $\delta V / V_{a}>$

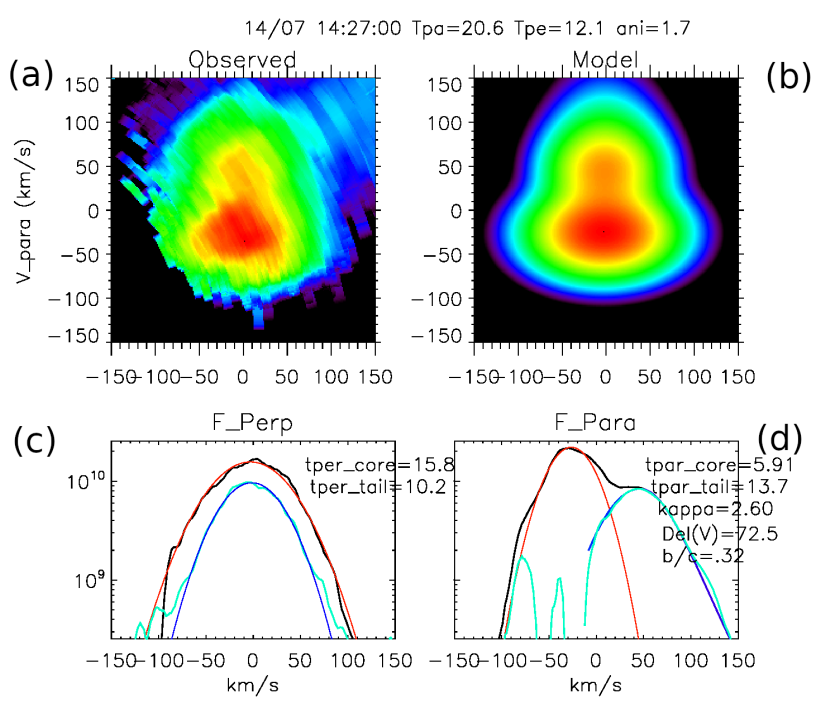

Fig. 4. Typical VDF and its model. Panel $a$ : slice of the measured VDF in a plane containing $B$, with parallel and perpendicular axis centered on the solar wind velocity. Panel $b$ : reconstructed VDF. Panels $c, d$ : plots of the distribution along the perpendicular and the parallel axis. The initial distribution is in black, the core bi-Maxwellian in red, the remaining population (initial minus core) in green, and the beam model in blue. The VDF unit is $\mathrm{s}^{3} \mathrm{~m}^{-6}$. The maximal VDF value is $2.510^{10}$.

$50 \%$ are observed in the form of oscillations with periods of a few tens of minutes as well as discontinuities and spikes at scales from a few minutes to a few seconds. The spectrum of fluctuations shows that the transition between the inertial domain (slope of -1.59 , on $B$ and $V$ ) and ion dissipation domain (slope of -3.8 for $B$ ) takes place at $\sim 0.5 \mathrm{~Hz}$ (i.e., 2-3 times the ion gyrofrequency). The cross-correlation of the components of $B$ and $V$, in each RTN direction, is observed down to this ion scale. The total anisotropy $\left(T_{t \|} / T_{t \perp}\right)$ fluctuates between $\sim 1$ and 3 , also on timescales ranging from several minutes to seconds. The interpretation of the total anisotropy and of its variations is the subject of the next section. This requires a focus on the detailed organization of the VDFs.

\section{Kinetic view of a slow Alfvénic flow: Examples of proton VDF}

A typical VDF and its model are shown in Fig. 4. It is measured at 14:27:00 when the total anisotropy is 1.7 , which is about its mean value for the whole time period studied here. The solar wind velocity $\left(V_{\mathrm{SW}}\right)$ is: $413,3.5,7.3 \mathrm{~km} \mathrm{~s}^{-1}$ and the magnetic field: $2.7,7.7,5.5 \mathrm{nT}$ (RTN). The density is $16.6 \mathrm{~cm}^{-3}$, the total parallel (perpendicular) temperature is $\sim 20.5 \mathrm{eV}(\sim 12.1 \mathrm{eV})$.

The measured VDF is represented in a plane which contains $B$ direction and $V_{\text {SW }}$ (Fig. 4a). The vertical and horizontal axis in the figure correspond, respectively, to the parallel $\left(V_{\|}\right)$and one of the perpendicular to $B\left(V_{\perp}\right)$ directions (the one perpendicular to both $B$ and $\left.V_{\mathrm{SW}}\right)$. The axes are centered on $\left(V_{\mathrm{SW}}\right)$. This $2 \mathrm{D}$ cut is obtained by integrating the sampling pixels on a slice of $\pm 30 \mathrm{~km} \mathrm{~s}^{-1}$ thickness from the $V_{\|}, V_{\perp}$ plane. This will be the case of all the distributions presented in the present article. In this plane, the pixels are generally portions of rings with radius determined by the measured energy and an orientation that depends on $\mathrm{B}$, with size $\sim 40 \times 20 \mathrm{~km} \mathrm{~s}^{-1}$ at energies of $\sim 1 \mathrm{keV}$.

This VDF is similar to those observed with Helios (Marsch et al. 1981, 1982). It composed of a dominant anisotropic core (red oval in the figure), such as $T_{\perp} / T_{\|}>1$, and a field-aligned 


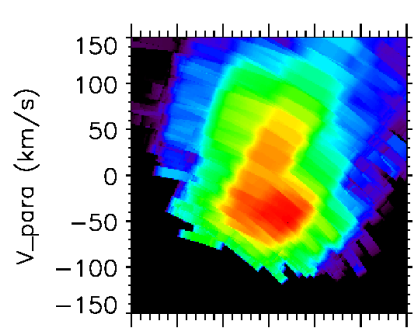

$-150100500 \quad 50100150$

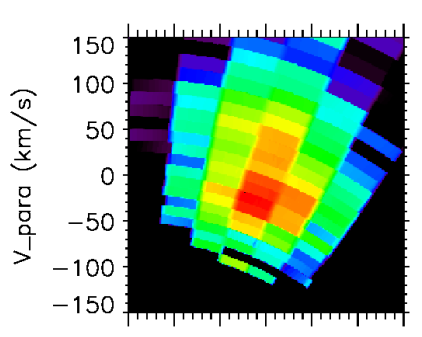

$-15010050050100150$
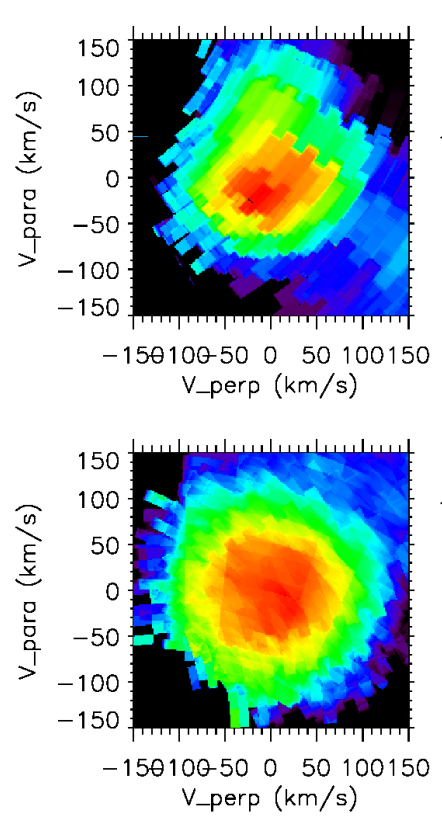

14/07 11:25:09 Tpa=34.4 Tpe=10.6 ani=2.74

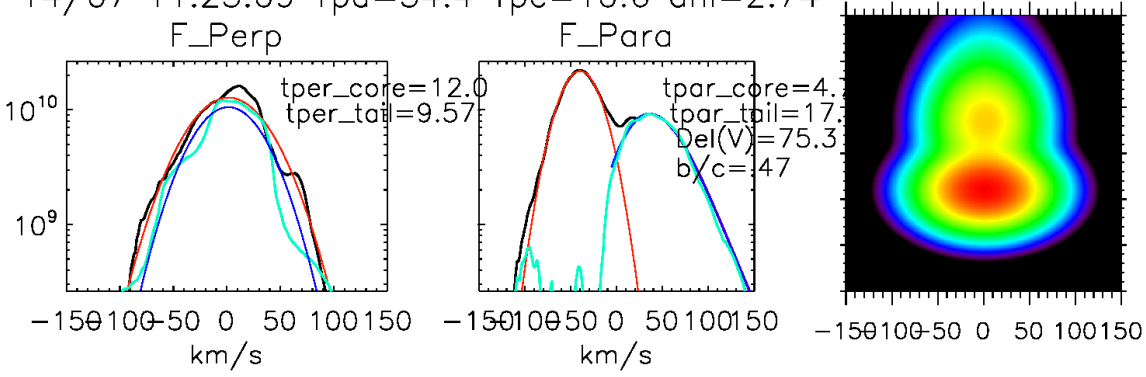

14/07 20:30:21 Tpa=24.3 Tpe=13.9 ani=1.7

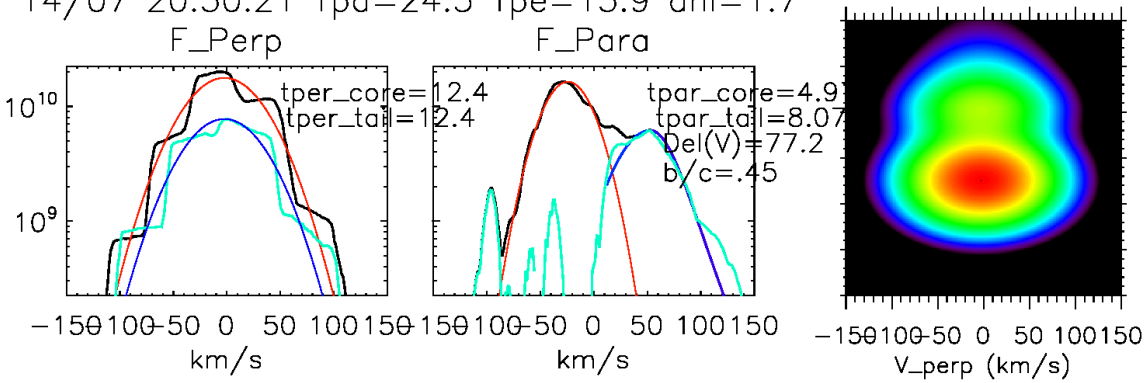

14/07 19:24:43 Tpa=14.06 Tpe=14.3 ani=0.98

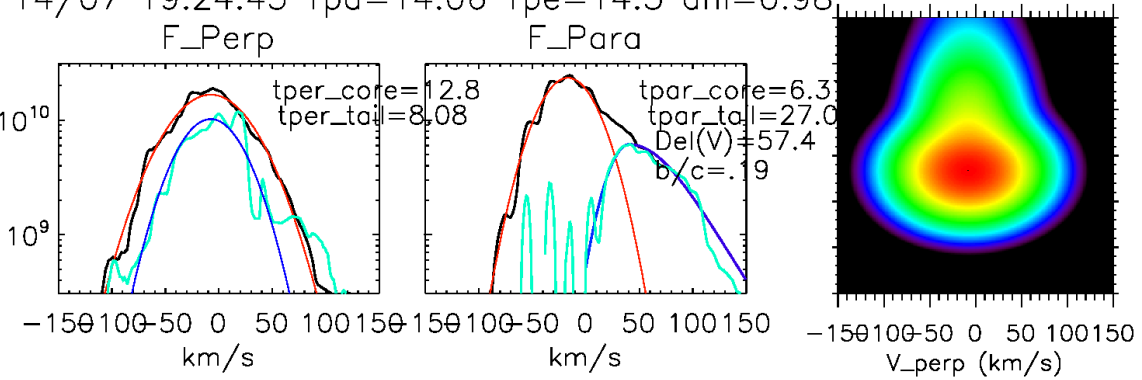

14/07 18:32:03 Tpa=18.1 Tpe=19.8 ani=0.96
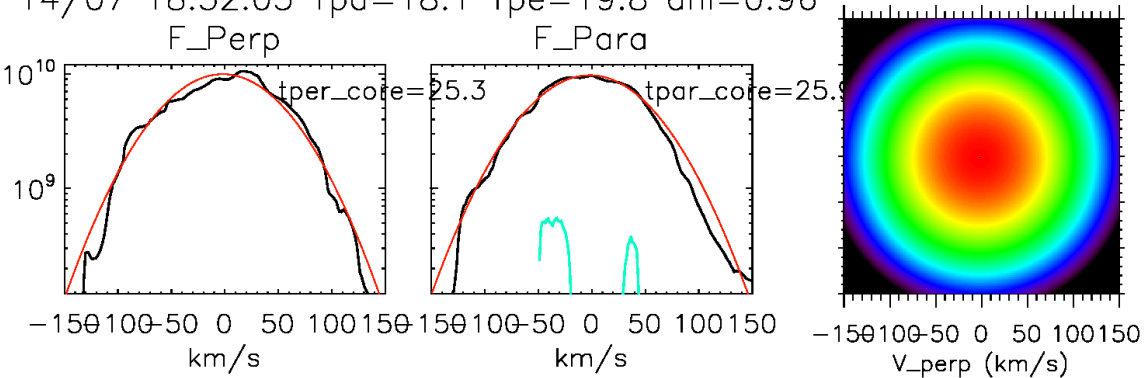

$(4)$

Fig. 5. Other examples of VDF corresponding to various total anisotropy. VDF 1, 2, and 3 provide further examples of core+beam distributions. The VDF 4 is an example of pure isotropic distribution. The VDF unit is $\mathrm{s}^{3} \mathrm{~m}^{-6}$. The color code is normalized to each distributions. From VDF 1 to 4 , the maximal VDF values are 2.6, 2.2, 3.08, and $1.010^{10} \mathrm{~s}^{3} \mathrm{~m}^{-6}$.

beam (orange extension along the $V_{\|}$axis). The fact that the total parallel temperature $\left(T_{t \|}\right)$ is larger than $\left(T_{t \perp}\right)$ is explained by the existence of the field-aligned beam. We note that alpha particles are virtually absent in this plot. Due to their $E / q=2$, they are indeed observed typically at 1.4 the proton velocity by PAS; thus, they lie far beyond the scope of the velocity plane considered here.

We modeled the distribution by a bi-Maxwellian core and an asymmetric beam in the parallel direction:

$$
\begin{aligned}
f\left(v_{\|}, v_{\perp}\right)= & A \exp \left(-\frac{\left(v_{\|}-V_{c}\right)^{2}}{k T_{c \|}}\right) \exp \left(-\frac{v_{\perp}^{2}}{k T_{c \perp}}\right) \\
& +B \exp \left(-\frac{v_{\perp}^{2}}{k T_{b \perp}}\right) F\left(v_{\|}-V_{b}\right),
\end{aligned}
$$

where $F\left(v_{\|}-V_{b}\right)$ is a Maxwellian distribution if $v_{\|}<V_{b}$ : $F=\exp \left(-\frac{\left(v_{\|}-V_{b}\right)^{2}}{k T_{b \|}}\right)$ or a kappa distribution if si $v_{\|}>V_{b}$ : $F=\left(1-\frac{\left(v_{\|}-V_{b}\right)^{2}}{\kappa W^{2}}\right)^{-1-\kappa}$.

Figures $4 \mathrm{c}$ and $\mathrm{d}$ show the plot of the measured VDF (in black) along the perpendicular and parallel axis. The biMaxwellian core is determined from a best fit (shown in red) around the peak of the distribution. This core fit is then removed from the total distribution (the resulting function is shown in green). We again modelled the resulting population in the vicinity of its maximum. It is generally close to a Maxwellian in the perpendicular direction and we thus determined the perpendicular temperature of the beam. In most cases, however, the beam is asymmetric in the parallel direction. It presents an abrupt cut 
towards low velocities and an extended tail toward large velocities. This high energy extension appears to be better fitted by the Kappa than Maxwellian distributions.

In the following, we will mostly consider the following parameters: (1) the parallel and perpendicular temperature of the core $\left(T_{c \|}\right.$ and $\left.T_{c \perp}\right),(2)$ same for the beam $\left(T_{b \|}\right.$ and $\left.T_{b \perp}\right)$, the parallel temperature here being the effective temperature deduced from the kappa distribution $\left(T_{b \|}=T_{\text {eff }}=\frac{m_{i} W^{2}}{2 k_{\mathrm{B}}} \frac{\kappa}{\kappa-1 / 2}\right)$, the drift speed between the core and the beam $\left(V_{b}-V_{c}\right)$, and the relative beam versus core density.

In the present case, we get $T_{c \|} \sim 5.9 \mathrm{eV}$ and $T_{c \perp} \sim 15.8 \mathrm{eV}$ and a core anisotropy of $\sim 3$. To remain consistent with past studies, we will systematically consider the $T_{c \perp} / T_{c \|}$ anisotropy for the core population. The drift speed is $\sim 72.5 \mathrm{~km} \mathrm{~s}^{-1}$ ( or $\sim 1.2 V_{a}$ ). The best fit is obtained with a kappa distribution of index 2.6, $T_{b \|} \sim 13.7 \mathrm{eV}, T_{b \perp} \sim 10.2 \mathrm{eV}$ and relative density between the beam and the core $\sim 0.32$. The beam thus represents one third of the total distribution and a significant part of the total proton population. Figure $4 \mathrm{~b}$ shows the reconstructed distribution.

Other examples of the VDF are shown in Fig. 5. We present three VDFs corresponding to total anisotropy varying from large to low values $(2.74,1.7,0.98$, from top to bottom, VDF 1 to $3)$. It is interesting to note that the cores present similar characteristics in the three cases with $T_{c \perp} \sim 12.0,12.4$ and $12.8 \mathrm{eV}$ and $T_{c \|} \sim 4.0,7.0$, and $6.3 \mathrm{eV}$. The $T_{c \perp} / T_{c \|}$ anisotropy thus varies from $\sim 3$ to $\sim 2$. The most important parameters that determines the variations of the total anisotropy are the beam characteristics and, in particular, its relative density. Here, it varies from 0.47 , 0.29 to 0.19 (VDF 1 to 3 ). Otherwise, the $T_{b \|}$ range from 8 to $19 \mathrm{eV}$ and $T_{b \perp}$ is generally close to $T_{c \perp}(\sim 12 \mathrm{eV})$. The beam drift is always $\sim 75 \mathrm{~km} \mathrm{~s}^{-1}$ (or $\sim 1.25 \mathrm{Va}$ ). We note that VDF 1 presents a bump in tail shape which appears to be relatively common when the total anisotropy is larger than 2.5. This suggests the possibility of triggering kinetic instabilities. We also note that observing a total anisotropy of $\sim 1$ does not mean that a Maxwellian distribution was measured. In this sense, VDF 3 can be considered as a typical VDF of low total anisotropy: the core is still notably anisotropic and a total anisotropy of $\sim 1$ is just a consequence of a particularly faint field-aligned beam, with typically a relative density below $\sim 0.2$.

Our observations of several tens of VDFs suggests that the core+beam VDF is the most common shape observed in this Alfvénic flow. They are systematically observed if $T_{t \perp}$ is smaller than $15 \mathrm{eV}$. However, as seen in Fig. 1, there are also time periods of greater $T_{t \perp}\left(T_{t \perp}>20 \mathrm{eV}\right)$. This often corresponds to intervals of total anisotropy of $\sim 1$ and this is when pure Maxwellians are often observed, with VDF 4 as a typical example. The Maxwellian fit is almost perfect, with same parallel and perpendicular temperature (25.9 and $25.5 \mathrm{eV}$ respectively). The existence of isotropic Maxwellian distributions may indicate that particular plasma processes act to relax the total anisotropy of the core+beam VDFs that represent the most common type of proton distribution in this Alvenic solar wind.

In the next sections, we present several structures associated with significant variations of the VDF shape. Examples of the creation of a strongly populated beam or, in reverse, of relaxation of the total anisotropy is also discussed.

\section{Case studies of structures associated with modifications of proton VDFs}

The first example of structure is a switchback seen around 19:25. The magnetic field and associated quantities are presented in

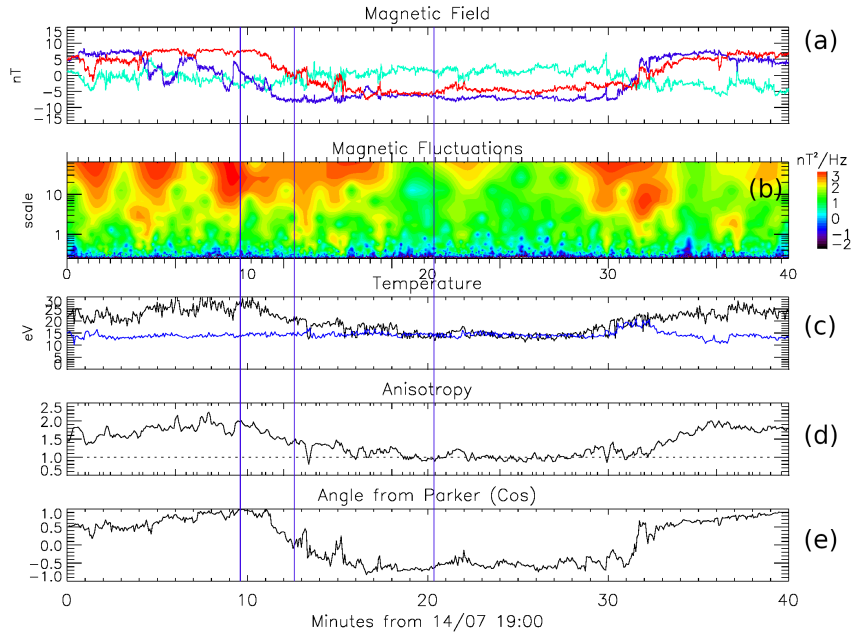

Fig. 6. Switchback observed from 19:10 to 19:32. Panel a: $B$ components ( $\mathrm{R}$ in red, $\mathrm{T}$ in blue, and $\mathrm{N}$ in green). Panel $b$ : wavelet transform of the magnetic fluctuation (sum of the power of each $B$ components, in $(\mathrm{nT})^{2} / \mathrm{Hz}$ ). Panel $c$ : total parallel (black) and perpendicular (blue) temperature $\left(T_{t \|}\right.$ and $\left./ T_{t \perp}\right)$. Panel $d$ : total anisotropy. Panel $e$ : cosine of the angle of $B$ with respect to the Parker spiral. The vertical lines indicates the time of measurements of the VDF presented in Fig. 7.

Fig. 6. The switchback is crossed from 19:10 (leading edge) to 19:30 (trailing edge). During this time period, the $B_{R}$ component is reversed (Fig. 6a), which is characteristic of switchbacks (Balogh et al. 1999; Horbury et al. 2018; Kasper et al. 2019; Dudok de Wit et al. 2020), with a negative angle when projected on the Parker spiral (Fig. 6d). The spiral angle is calculated considering a solar wind speed of $450 \mathrm{~km} \mathrm{~s}^{-1}$. The associated global modifications of the proton population can be seen in Fig. 1. The switchback corresponds to an increase in the average energy of the proton population, from $\sim 950$ to $\sim 1050 \mathrm{eV}$, with a variation of flux by a factor of $\sim 2$. The $V_{R}$ also increases by $\sim 60 \mathrm{~km} \mathrm{~s}^{-1}$, as the density, from 13 to $17 \mathrm{~cm}^{-3}$.

Figure $6 \mathrm{~b}$ shows the magnetic spectral power, computed as the sum of the power density of each magnetic component (obtained from a wavelet transform). It indicates that the switchback itself corresponds to a quieter state of plasma than its environment. The spectral power at scales ranging from $0.25 \mathrm{~s}$ to $60 \mathrm{~s}$ decreases by two orders of magnitude inside the structure, from 19:14 to 19:29. The maximal spectral power is thus located at the edges of the structure and in its immediate vicinity. Figures $6 \mathrm{c}$ and $\mathrm{d}$ present the total parallel and perpendicular temperatures and the total anisotropy. The internal part of the switchback corresponds to a complete relaxation of the total temperature anisotropy. While $\left(T_{t \|} / T_{t \perp}\right)$ is $\sim 2$ before and after the switchback, it is $\sim 1$ inside the structure. It is interesting to note that the low magnetic spectral power inside the switchback also corresponds to the relaxed total anisotropy.

In Fig. 7, three VDF measured before (19:09:36 VDF 1), during the transition (19:12:31 VDF 2) and in the center of the structure (19:20:20 VDF 3) are presented. VDF 1 is measured when the spectral density of the magnetic fluctuations is at maximum. It is characterized by a core with a $T_{c \perp} / T_{c \|}$ anisotropy of $2.5\left(T_{c \perp} \sim 14 \mathrm{eV}, T_{c \|} \sim 5.7 \mathrm{eV}\right)$ and a rather dense beam (relative density of 0.63 , velocity shift of $65 \mathrm{~km} \mathrm{~s}^{-1}$, and effective temperature of $18 \mathrm{eV})$. By contrast, the VDF 3 is characterized by a less anisotropic core $\left(T_{c \perp} \sim 15.7 \mathrm{eV}, T_{c \|} \sim 8.3 \mathrm{eV}\right.$, anisotropy of $\left.\sim 1.9\right)$ and a fainter beam (relative density of 0.16). The transition from the external anisotropic to the internal isotropic plasma is thus 


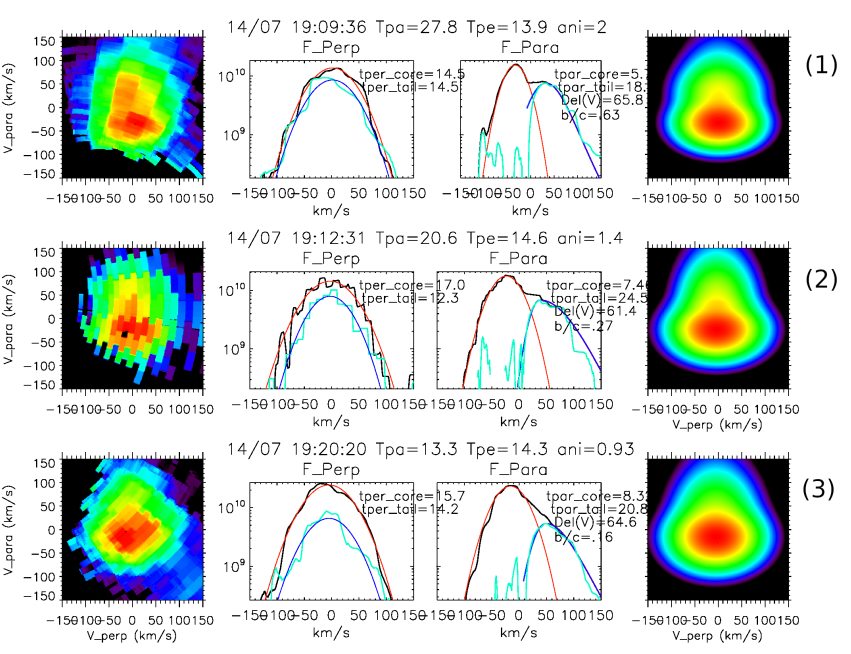

Fig. 7. Measured VDF and models before (1), at the transition (2), and inside (3) the switchback. From VDFs 1-3, the respective maximal VDF values are 1.83, 2.07, and $2.6610^{10} \mathrm{~s}^{3} \mathrm{~m}^{-6}$ ).

mostly associated with a decrease of the beam relative density, by a factor of $\sim 4$. The VDF 2 is measured at the transition. It presents somewhat intermediate characteristics between VDF 1 and 3, albeit with a decrease of the beam relative density (0.27). An interesting feature of VDF2 is the apparent D-shape structure of its core. This is generally considered as a consequence of magnetic reconnection (see also, Fedorov 2021, for evidence of reconnection linked to switchback and other examples of a D-shaped VDF).

This switchback can be considered as an example of structure that relaxes the total anisotropy of the flow. Core+beam VDFs are observed both in the immediate vicinity and inside the switchback, but the relative density of the beam has strongly decreased in the structure (a factor of $\sim 4$ here), which corresponds to a decrease of the total anisotropy from $\sim 2$ to 1 . This also coincides with a decrease of the $T_{c \perp} / T_{c \|}$ anisotropy of the core, from 2.5 to 1.9. Further cases of switchback must be investigated to confirm this observation of total anisotropy relaxation. A recent paper (Woodham et al. 2021), using SPAM measurements onboard PSP, shows also an example of more isotropic core in switchback.

Our second example illustrates the reverse situation. It corresponds to the crossing of a current sheet during which a sudden and sharp increase of the total anisotropy is observed. The general context is described in Fig. 8, where $10 \mathrm{~min}$ of data are presented (10:40-10:50). The structure of interest is a sharp current sheet crossed at 10:46:45, corresponding to a sharp discontinuity of $B_{N}$ (by $10 \mathrm{nT}$ ), $B_{R}$ showing a smoother increase, from $10: 45: 45$ to $10: 46: 45$. Before the current sheet crossing, from $10: 43: 00$ to $10: 46: 30, B_{R}$ is slightly negative and the angle from the Parker spiral is close to $90^{\circ}$. This time interval thus corresponds to a strongly inclined magnetic field with respect to the radial direction. It is also associated with a large power of the magnetic fluctuations which are about three orders of magnitude larger than elsewhere. The power is maximized at the edge of the structure (10:46:45) and locally reaches $10^{3} \mathrm{nT}^{2} \mathrm{~Hz}^{-1}$ at $1 \mathrm{~Hz}$. This is one of the largest power spectral density measured at scales close of the gyroperiod for the entire 20 hour period. The corresponding waves can be seen on $B_{T}$ and $B_{N}$, just after the crossing of the current sheet. The interesting observation is that this coincides with a time period lasting $\sim 1$ min of very large total anisotropy. Before 10:45:30, the total anisotropy is

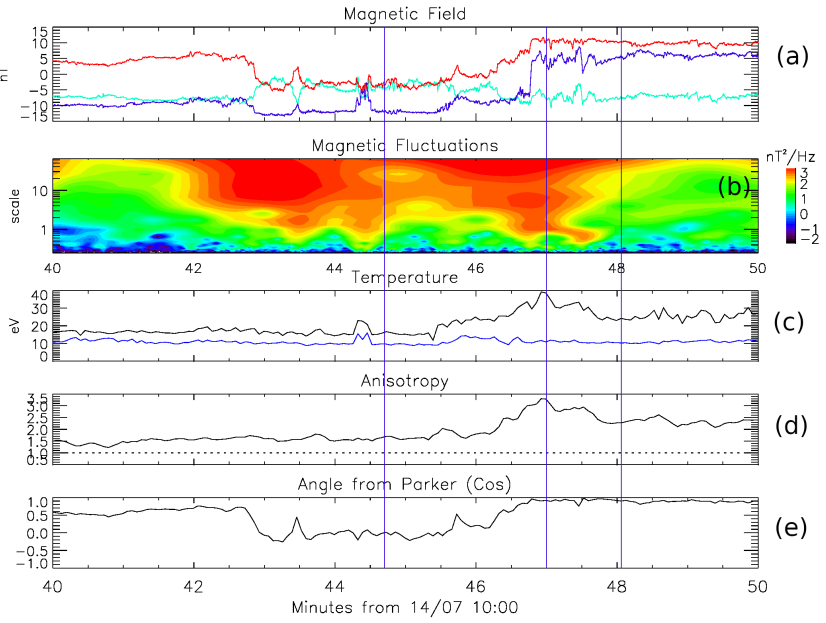

Fig. 8. Current sheet associated with a strong increase of the total anisotropy. Same panels as in Fig. 7.
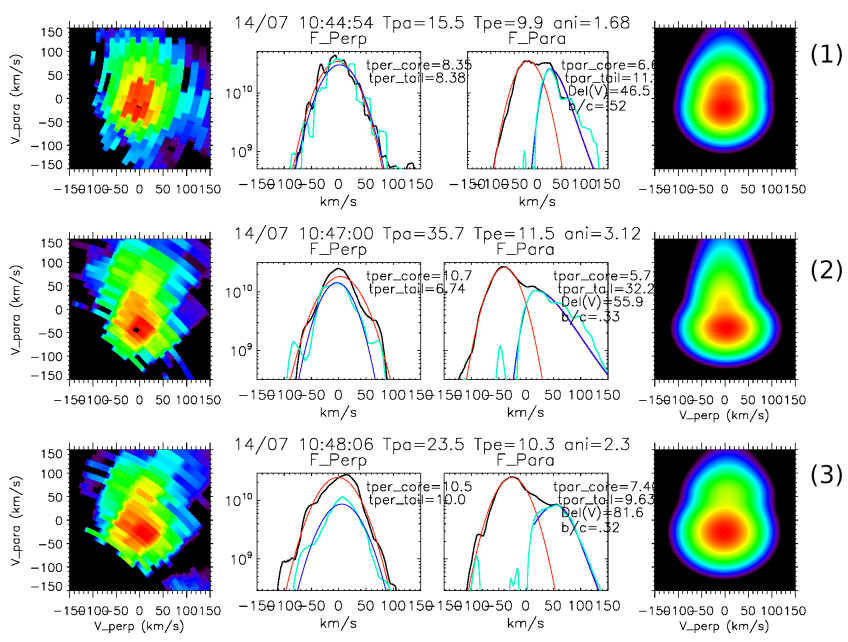

Fig. 9. Measured VDF and models before (1), at the maximal total anisotropy (2) and after (3) the current sheet.From VDF 1 to 3, the maximal VDF values are 5.1, 3.2, and $2.910^{10} \mathrm{~s}^{3} \mathrm{~m}^{-6}$ ).

$\sim 1.3-1.5$, corresponding to $T_{t \|} \sim 15-17 \mathrm{eV}$ and $T_{t \perp} \sim 10-12 \mathrm{eV}$. The anisotropy strongly increases from 10:46 to 10:47, when a maximum of 3.4 is reached $\left(T_{t \|} \sim 40 \mathrm{eV}\right)$. About 1 minute later (10:47:45), the total anisotropy decreases to below 2.5 and a more classical situation is recovered.

In Fig. 9, we present three VDFs measured before the current sheet (10:44:54 VDF 1), when the total anisotropy is at maximum (10:47:00 VDF 2) and after the crossing (10:48:06 VDF 3 ). The VDF 1 is measured when the magnetic field is quasi perpendicular to the radial direction. It is characterized by a double bump in the parallel direction, which is best described by the juxtaposition of a core and a dense and rather cold beam $\left(T_{b \|} \sim 11 \mathrm{eV}\right.$, relative density 0.5$)$. The drift speed is unusually small $\left(46 \mathrm{~km} \mathrm{~s}^{-1}\right)$ and both the core and the beam are almost isotropic. VDF 2 is characterized by a very developed beam, with $\left(T_{b \|}=32 \mathrm{eV}\right.$, drift speed $\sim 55 \mathrm{~km} \mathrm{~s}^{-1}$, and relative density $=0.33$. The strong heating of the beam in the parallel direction explains the very strong total anisotropy. VDF 3 still presents a large total anisotropy (2.3). The beam is still relatively dense (0.32) and rather cold $\left(T_{b \|} \sim 9.6 \mathrm{eV}\right)$. It presents a large drift speed $\left(82 \mathrm{~km} \mathrm{~s}^{-1}\right)$. Over the whole sequence, the 

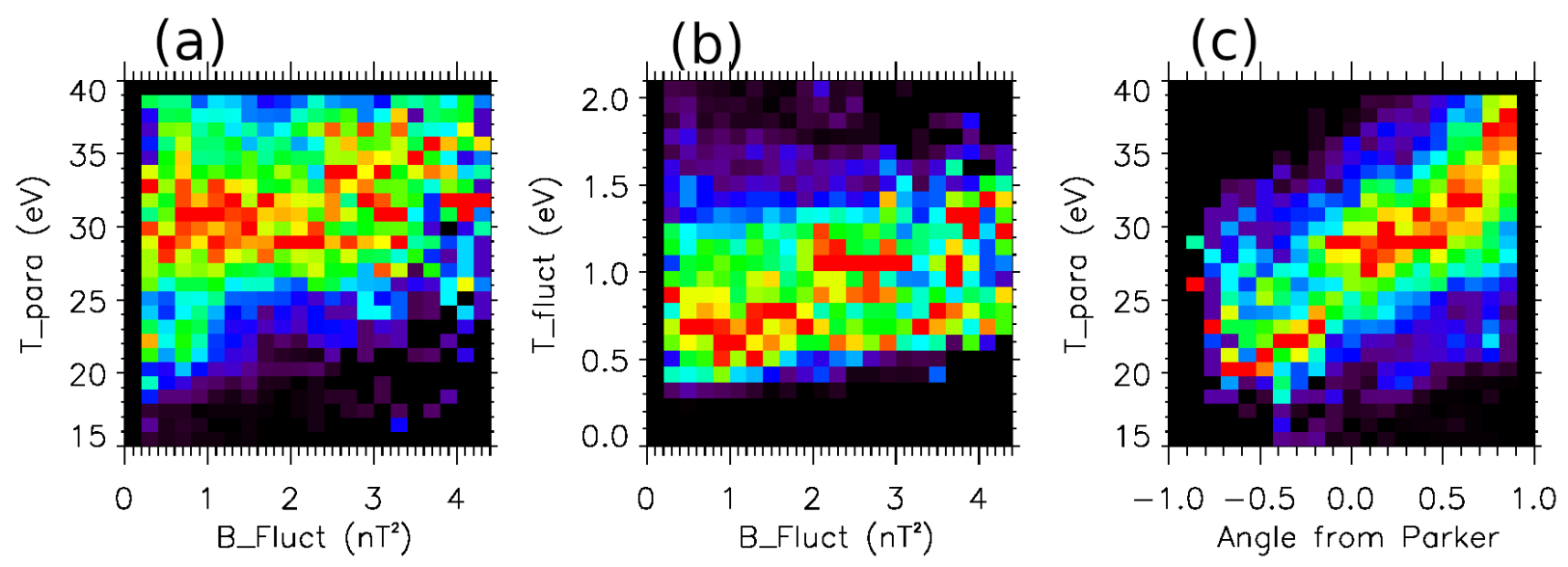

Fig. 10. Statistical analysis of the possible links between the kinetic properties of the flow (using $T_{t \|}$ as a proxy) and the amplitude of the $B$ fluctuations (panels $a$ and $b$ ) and $B$ direction from Parker spiral (panel $c$ ). The cosine of the angle is considered (1: aligned outward directed, 0: perpendicular, -1: anti-aligned). A normalization was performed for each interval of fluctuations or angle, so that the pixel values vary from 1 (red) to 0 (black).

characteristics of the core remain relatively stable, with a modest anisotropy $\sim 1.3-2$, corresponding to $T_{c \|} \sim 6-7.5 \mathrm{eV}$ and $\left.T_{c \perp} \sim 8-11 \mathrm{eV}\right)$.

In summary, this sharp current sheet - corresponding to a transition between a region of quasi-perpendicular to quasiparallel B (with respect of the radial direction) - is associated with a strong increase of total anisotropy that reaches $\sim 3$ for about one minute. This is the result of the formation of a dense and hot beam just in the vicinity of the discontinuity. This precisely occurs when the spectral power of the magnetic fluctuations is maximal and extends down to the gyroperiod. By contrast, the core population remains relatively stable. It is obviously tempting to consider that processes occurring at the current sheet are responsible for the evolution of the beam shape and, in particular, of its heating (Sorriso-Valvo et al. 2019). Is this mediated by the wave activity seen at the gyroperiod or is the wave activity triggered by kinetic instabilities associated with the developed beam are open questions.

\section{Discussion and conclusions}

A first conclusion of this work is to show that the remarkable correlation between the $\mathbf{B}$ and $\mathbf{V}$ components is observed down to the sub-ion domain, at timescales of seconds. This concerns a wide variety of high amplitude fluctuations, such as $\Delta B /|B|>0.5$ and $\Delta V / V_{a}>0.5$ : long period oscillations (a few tens of minutes), isolated peaks, and current sheets crossed in a few seconds.

A second outcome of this work is the capability to document the broad variability of the shape of the proton VDFs in an Alfvénic flow. If the VDFs appear very systematically as the juxtaposition of a core and a field-aligned beam, which has already been described from Helios observations, the relative density of the beam, its drift speed as $T_{b \|}$ can considerably change at scales as short as as a few seconds. By comparison, the characteristics of the core are more stable. In general, we can consider that the variations in the beam characteristics largely explain the variations in $T_{t \|}$ (total temperature) and, therefore, in the total anisotropy. In other words, a strong total anisotropy (typically larger than 2) can be considered a proxy for the existence of a well-developed field-aligned beam (typically with relative density $>0.3$, drift speed $>60 \mathrm{~km} \mathrm{~s}^{-1}$, and $T_{b \|}>15 \mathrm{eV}$ ). However, in reverse, a total anisotropy $\sim 1$ is not an indication of Maxwellian plasma. The anisotropic core is indeed present in a majority of cases but the beam is simply fainter. There are time periods when the proton VDF is really isotropic and these are associated with a $T_{c \perp}$ rise, becoming close to $20 \mathrm{eV}$ compared to $12-15 \mathrm{eV}$ as commonly measured. This may indicate that efficient heating processes have relaxed or are relaxing the total anisotropy. We also analyzed two cases of magnetic structures that are associated with significant changes in the shape of VDFs, one corresponding to relaxation of total anisotropy (a switchback), the other, on the contrary, to its strong increase (a current sheet). In both cases, what has particularly evolved are the characteristics of the beam. It becomes tenuous inside a switchback and conversely, dense and particularly extended towards large parallel velocities in the immediate vicinity of the current sheet.

It seems clear that VDFs able to drive different kinds of kinetic instabilities are observed during this episode of slow wind. The VDF 1 in Fig. 5 provides an example of core + welldeveloped beam with a positive slope of the distribution with respect to $V_{\|}$and a core-beam drift larger than $V_{a}$. According to numerous studies (see reviews by Gary 1993; Marsch 2006; Verscharen et al. 2019), this may drive various ion-ion instabilities and, in particular, amplify ion-cyclotron waves. VDF 2 in Fig. 9, with a total $T_{\perp} / T_{\|}$anisotropy $>3$, is representative of another kind of distribution that may drive instabilities. At this time, the plasma regime is indeed such that $\beta_{\|}>2$ (ratio between $P_{\|}$and magnetic pressure), which is compatible with the onset of a firehose instability (Hunana et al. 2019). Obviously, a detailed comparison with the waves is needed to establish that these different forms of instabilities may, at least occasionally, developed. One difficulty that we would point out is the pronounced variability of the proton VDFs in this turbulent flow. They indeed show significant changes in their characteristics on timescales close to the expected e-folding time of the various instabilities. The question of how classical theories of kinetic instabilities apply in these conditions arises at this point.

Considering that the magnetic structures propagating in this Alfvénic flow could coincide with significant modifications in the proton VDF shape, it is obviously tempting to find out whether this possibility could result from a simple characteristic of the structures themselves or of the observed magnetic turbulence. We attempted multiple forms of correlation for this purpose. For a first approach, we simply considered $T_{t \|}$ as a proxy for the characteristics of the beam. We show three examples 
of such correlations in Fig. 10, in the form of two-dimensional histograms that take into account the 20 hours of observations described here.

A first idea is to examine whether there is a link between $T_{t \|}$ and the level of magnetic fluctuations. This level is obtained by integrating the spectral density of the magnetic fluctuations (deduced from waveform transform) in the range of $0.25-60 \mathrm{~s}$. Since the statistics of the magnetic fluctuations greatly vary, the histogram is normalized for each interval of fluctuation amplitude. As seen in Fig. 10a, there is (possibly!) a slight tendency for $T_{t \|}$ to increase with the amplitude of fluctuations, but it is clear that this is not systematic. At best, $T_{t \|}$ is poorly controlled by the amplitude of the magnetic fluctuations. In other words, if we come back to the kinetic properties of the proton population and consider $T_{t \|}$ as a proxy for beam characteristics, situations of well-developed or, on the contrary, faint beams are encountered both in the presence of strong or weak magnetic fluctuations.

A second possibility is considers whether short-scale temperature variations can be related to the amplitude of the magnetic fluctuations. If time intervals of a certain duration ( 2 min chosen in this case) are set, we explore whether a correlation between the variability of $T_{t \|}$ on the interval (so, of the beam characteristics) and the level of the magnetic fluctuations exists. This is shown in Fig. 10b. The trend appears to be clearer than before: $T_{t \|}$ is exhibits more fluctuation in the presence of large magnetic fluctuations. In other words, the level of turbulence increases the variability of the beam characteristics.

The most spectacular correlation is obtained by considering the direction of $B$ (Fig. 10c). It shows that a large $T_{t \|}$ is much more probable when the field is aligned with the Parker spiral. For large deviations of $B$ from the Parker spiral, a fortiori for reversals, there is no observation of $T_{t \|}>20 \mathrm{eV}$. We note that this analysis is done as $B$ is generally directed outward and, thus, that reversals have to be considered as perturbations of an averaged field pointing outward. Using, again, $T_{t \|}$ as a proxy of the beam characteristics, the immediate conclusion is that situations where $B$ is aligned with the Parker spiral (or quasi-radial $B$ ) favor the existence of strongly developed beams. Statistically, there would be a direct link between the kinetic characteristics of this type of solar wind and the local $B$ direction. Flux tubes oriented radially would contain proton VDF with well-developed beams when inclined or strongly deviated flux tubes might contains more isotropic VDFs. Obviously, this link between the local orientation of the flux tube and the kinetic properties of its contain should be confirmed by the quantitative analysis of much more VDFs (and not only using $T_{t \|}$ as a proxy).

In reporting on the first measurements made by PAS, our general conclusion is that these observations show the variability of the kinetic properties of the proton VDFs in an Alfénic flow, and the possible strong effects of magnetic structures on their evolution. They also show the capabilities of PAS to provide the required measurements to progress in these areas of study, thanks to its precision and its ability to measure 3D VDF at a sub-second cadence.

Acknowledgements. P. L. would like to thank all the outstanding women and men who have made PAS, SWA and Solar Orbiter a reality, including the persons who contribute to the development of software used for the analysis (CL, AMDA, and CDPP). The French part of this work is supported by CNES. Solar Orbiter SWA work at UCL/MSSL is currently funded under STFC grants ST/T001356/1 and ST/S000240/1. The work of L.P. was supported by the Czech Science Foundation, grant no. 19-18993S. D. V. is supported by STFC Ernest Rutherford Fellowship ST/P003826/1 and STFC Consolidated Grant ST/S000240/1. Solar Orbiter is a space mission of international collaboration between ESA and NASA, operated by ESA.

\section{References}

Alexandrova, O., Saur, J., Lacombe, C., et al. 2009, Phys. Rev. Lett., 103, 165003 Bale, S. D., Badman, S. T., Bonnell, J. W., et al. 2019, Nature, 576, 237

Balogh, A., Forsyth, R. J., Lucek, E. A., Horbury, T. S., \& Smith, E. J. 1999, Geophys. Rev. Lett., 26, 631

Belcher, J. W., \& Davis Jr., L. 1971, J. Geophys. Res., 76, 3534

Belcher, J. W., Davis Jr., L., \& Smith, E. J. 1969, J. Geophys. Res., 74, 2302

Borovsky, J. E. 2016, J. Geophys. Res. (Space Phys.), 121, 5055

Bruno, R., \& Carbone, V. 2013, Liv. Rev. Sol. Phys., 10, 2

Bruno, R., Bavassano, B., \& Villante, U. 1985, J. Geophys. Res., 90, 4373

Cuperman, S., Weiss, I., \& Dryer, M. 1983, ApJ, 273, 363

D’Amicis, R., Bruno, R., \& Bavassano, B. 2011, J. Atm. Sol.-Terr. Phys., 73, 653

D'Amicis, R., Matteini, L., \& Bruno, R. 2019, MNRAS, 483, 4665

D’Amicis, R., Bruno, R., Panasenco, O., et al. 2021, A\&A, 656, A21 (SO Cruise Phase SI)

Demars, H. G., \& Schunk, R. W. 1990, Planet Space Sci., 38, 1091

Dudok de Wit, T., Krasnoselskikh, V. V., Bale, S. D., et al. 2020, ApJS, 246, 39

Fedorov, A., Louarn, P., Owen, C. J., et al. 2021, A\&A, 656, A40 (SO Cruise Phase SI)

Fox, N. J., Velli, M. C., Bale, S. D., et al. 2016, Space Sci. Rev., 204, 7

Gary, S. P. 1993, Theory of Space Plasma Microinstabilities

Génot, V., Budnik, E., Jacquey, C., et al. 2021, Planet. Space Sci., 201, 105214

Gershman, D. J., Dorelli, J. C., F.-Viñas, A., \& Pollock, C. J. 2015, J. Geophys. Res. (Space Phys.), 120, 6633

Horbury, T. S., Matteini, L., \& Stansby, D. 2018, MNRAS, 478, 1980

Horbury, T. S., O’Brien, H., Carrasco Blazquez, I., et al. 2020, A\&A, 642, A9

Hunana, P., Tenerani, A., Zank, G. P., et al. 2019, J. Plasma Phys., 85, 205850602

Hundhausen, A. J. 1970, Rev. Geophys. Space Phys., 8, 729

Kasper, J. C., Bale, S. D., Belcher, J. W., et al. 2019, Nature, 576, 228

Leubner, M. P. 2004, Phys. Plasmas, 11, 1308

Maksimovic, M., Pierrard, V., \& Riley, P. 1997, Geophys. Rev. Lett., 24, 1151

Marsch, E. 2006, Liv. Rev. Sol. Phys., 3, 1

Marsch, E., Rosenbauer, H., Schwenn, R., Muehlhaeuser, K. H., \& Denskat, K. U. 1981, J. Geophys. Res., 86, 9199

Marsch, E., Schwenn, R., Rosenbauer, H., et al. 1982, J. Geophys. Res., 87, 52

Matteini, L., Landi, S., Hellinger, P., \& Velli, M. 2006, J. Geophys. Res. (Space Phys.), 111, A10101

Matteini, L., Hellinger, P., Goldstein, B. E., et al. 2013, J. Geophys. Res. (Space Phys.), 118, 2771

Moore, T. E., Pollock, C. J., \& Young, D. T. 1998, Washington DC American Geophysical Union Geophysical Monograph Series, 102, 105

Müller, D., St. Cyr, O. C., Zouganelis, I., et al. 2020, A\&A, 642, A1

Nicolaou, G., Livadiotis, G., \& Wicks, T. 2020, Entropy, 22, 212

Owen, C. J., Bruno, R., Livi, S., et al. 2020, A\&A, 642, A16

Pierrard, V., \& Lazar, M. 2010, Sol. Phys., 267, 153

Roberts, D. A., Goldstein, M. L., Klein, L. W., \& Matthaeus, W. H. 1987, J. Geophys. Res., 92, 12023

Sahraoui, F., Goldstein, M. L., Robert, P., \& Khotyaintsev, Y. V. 2009, Phys. Rev. Lett., 102, 231102

Sorriso-Valvo, L., Catapano, F., Retinò, A., et al. 2019, Phys. Rev. Lett., 122, 035102

Suess, S. T. 2007, in Second Solar Orbiter Workshop, eds. E. Marsch, K. Tsinganos, R. Marsden, \& L. Conroy, ESA SP, 641, 11

Tu, C. Y., \& Marsch, E. 1995, Space Sci. Rev., 73, 1

Verscharen, D., Klein, K. G., \& Maruca, B. A. 2019, Liv. Rev. Sol. Phys., 16, 5

Viall, N. M., \& Borovsky, J. E. 2020, J. Geophys. Res. (Space Phys.), 125, e26005

von Steiger, R. 2008, in The solar wind throughout the solar cycle, eds. A. Balogh, L. J. Lanzerotti, \& S. T. Suess, 41

Whang, Y. C. 1971, J. Geophys. Res., 76, 7503

Woodham, L. D., Horbury, T. S., Matteini, L., et al. 2021, A\&A, 650, L1

Zouganelis, I., De Groof, A., Walsh, A. P., et al. 2020, A\&A, 642, A3 